\title{
Root and Canal Configuration of Mandibular First Molars in a Yemeni Population: A Cone-beam Computed Tomography
}

\author{
DIham M. SENAN, (1) Ahmed A. MADFA, (1) Hatem A. ALHADAINY
}

\section{ABSTRACT}

Objective: To describe root and canal morphology of mandibular first molars (MFMs) in a Yemeni population using cone-beam computed tomography (CBCT).

Methods: CBCT images of 500 right and left untreated MFMs with fully developed roots from 250 Yemenis (125 male and 125 female) comprised the sample size of this study. The following characteristics were recorded: (1) number of roots and their type and morphology, (2) number of canals orifices per root, (3) type of canal configuration and (4) primary variations in the morphology of the root and canal systems.

Results: $96.8 \%$ of MFMs are double-rooted. A third root was found in $3.2 \%$, more in females than males. Mesial root was mainly ribbon-shaped $(92.2 \%)$ and distal root was kidney-shaped in $56.2 \%$. Two canals orifices were found in mesial root of $95.8 \%$ and one canal orifice was found in distal root of $96.4 \%$. Vertucci type II canal configuration was the most frequent $(57 \%)$, followed by type IV $(35.6 \%)$ in mesial root. Type III canal configuration was the most prevalent (48.8\%), followed by type I (41\%) in distal root. Variant 3 represented the most common root and canal morphology (89.8\%).

Please cite this article as: Senan EM, Madfa AA, Alhadainy HA. Root and Canal Configuration of Mandibular First Molars in a Yemeni Population: A Cone-beam Computed Tomography. Eur Endod J 2020; 1: 10-7

From the Department of Restorative and Prosthodontic (E.M.S.凶elhamdent06@yahoo. com, e.senan@ust.edu, A.A.M.) College of Dentistry, University of Science and Technology, Sana'a, Yemen; Department of Conservative Dentistry (A.A.M.), Faculty of Dentistry, Thamar University, Dhamar, Yemen; Department of Restorative Dental Sciences (A.A.M.), College of Dentistry, Hail University, Hail, Saudi Arabia; Department of Endodontics (H.A.A.), Faculty of Dentistry, Tanta University, Tanta, Egypt; Department of Endodontics (H.A.A.), College of Dentistry, University of North Carolina, Chapel Hill, USA

Received 08 July 2019,

Accepted 28 January 2020

Published online: 11 March 2020 DOI 10.14744/eej.2020.99609

This work is licensed under a Creative Commons Attribution-NonCommercial 4.0 International License.
Conclusion: MFMs in Yemeni population are mainly two-rooted with 3.2\% having a supernumerary distolingual root. Cross section of mesial root was mainly ribbon-shaped and distal root was kidney-shaped. Vertucci type II and III configurations were the higher incidence in mesial and distal roots, respectively. The presence of two canals in mesial root and one canal in distal root of MFMs with two separate roots (variant 3) was the most common morphology.

Keywords: Canal anatomy, cone beam computed tomography, mandibular first molar, Yemeni population

\section{HIGHLIGHTS}

- Even if they occur in small percentages, anatomical variations of mandibular first molars roots and canals must be kept in mind and looked for while treating such teeth.

- Careful reading of CBCT images (whenever needed) is essential to reveal the complex internal pulp anatomy to prevent any missed canals and subsequent undesirable outcomes.

- Awareness of morphological differences of mandibular first molars in Yemeni population with other different populations increases the rate of endodontic treatment success of these teeth.

\section{INTRODUCTION}

Proper diagnosis followed by complete biomechanical cleaning and shaping and three-dimensional obturation of root canal system are essentials for long term predictable endodontic outcomes (1). To increase endodontic success rate, sufficient knowledge of the root canal system and its possible variations among different populations is essential in terms of canal numbers, types and orientation (1, 2). Insufficient or lack of information in this aspect may lead to a missed canal which ends in endodontic failure due to the harmful effects of the micro-organisms and infected pulp tissues (2).
Several studies have examined mandibular first molars (MFMs) in different populations and reported a number of anatomical variations and anomalies related to their roots and canal systems $(1,3,4)$. Typically, MFMs have two roots, (mesial and distal roots), but an additional third root may exist. When this third root is located in the distolingual (DL) side, it is called radix Entomolaris (RE), but if it is located in the mesiobuccal (MB) side, this is called radix paramolaris (RP). RE is one of the most frequent variations in number of roots which is coronally attached to the distal root and may 
be apically partially fused with or separated from other roots (5). RE is usually short and small but it may reach the length of adjacent roots (5), and its prevalence was found to be $10-29 \%$ in Eastern Asian populations (1, 4, 6, 7), 0.7-2.67\% in European populations $(8,9), 6.16 \%$ in Southern American population (8), $3.1 \%$ in African populations (10), $6 \%$ in Arabian population (11), and $2.06 \%$ in a Turkish population (12). This anatomical variation represents challenges in endodontic treatment as the orifice of its canal is not easily located and may lead to subsequent endodontic problems related to a missed root canal (13). $\mathrm{RP}$ is a rare anatomical variation with less frequency than the RE. Its dimensions can be variable from just a short conical root extension to a full length mature root which could be separate or fused with the main root (5).

Another frequent variation revealed in the studies of MFMs is related to different configurations of their roots and canal types. Mesial root was found to have Vertucci type IV as a dominant type in many populations $(10,12,14-18)$ while others showed Vertucci type II to be the dominant type $(19,20)$, whereas distal root showed Vertucci type I as the most prevalent configuration in most populations $(8,10,12,14-18)$.

Several methods have been used for studying root configuration and canal morphology of human teeth in various populations. Cone beam computed tomography (CBCT) digital imaging has been reported to be a common in vivo diagnostic aid in clinical practice that showed a better detection of Vertucci type I canal and a similar precise detection of Vertucci type II canals when compared with the in vitro clearing method while using micro-computed tomography (micro-CT) imaging as the reference standard for both methods (21). The CBCT can provide accurate images of high diagnostic quality, with lower radiation dose and shorter scanning times (22). In addition, it is an available and less expensive method that can be used in daily dental practice and for in vivo studies (6).

Despite the lack of nationwide database in Yemen on the prevalence of dental caries, a study reported higher prevalence of dental caries among Yemeni population compared to other countries which indicates a high demand for endodontic therapy in Yemenis (23). Literature search reveals comparatively a few numbers of studies in root configuration and canal anatomy of MFMs in the middle east populations $(10,11,20,24)$. In addition, no published study has been conducted yet that investigated root and canal morphology of MFMs in Yemeni population. Therefore, this study aimed to investigate root and canal morphology of MFMs in a Yemeni population using CBCT.

\section{MATERIALS AND METHODS}

\section{Study design}

The protocol of this descriptive observational cross-sectional study was approved by the Medical Ethics Committee of University of Science and Technology (UST), Sana'a, Yemen. Written and verbal consents were obtained from all individuals included in the study.

\section{Study population}

This study was conducted in dental polyclinics of College of Dentistry of UST, Sana'a, Yemen. This UST dental college attracts a huge number of varieties of patients who come from Sana'a and its surroundings governorates. This large city is the capital of Yemen and majority of its residents moved from all over Yemen.

The study sample consisted of dental patients (18-40 years), referred for treatment to dental polyclinics at the UST dental college. They were examined during the period from August 2016 until July 2018. The inclusion criteria comprised individuals with bilateral fully erupted permanent MFMs with mature completely formed roots and sound crown or with initial noncavitation caries. Individuals were excluded from the study if they had MFMs with open apices, root resorptions, calcifications, fractures, previous endodontic treatment, posts, or crown restorations.

After screening for selection according to the inclusion/exclusion criteria, CBCT images of 500 MFMs (right and left) from 250 Yemenis ( 125 male and 125 female) were collected to represent the final sample size.

\section{CBCT imaging}

All scans followed manufacturer's recommendation protocol. CBCT images were taken using Pax-Flex3D imaging system (VATECH Global, Hwaseong-si, Korea) operated at 50-90 kVp and 2-10 mA, with a field of view (FOV) of $50 \times 50 \mathrm{~mm}$, an exposure time of 15-24 seconds and a voxel size of $120 \mu \mathrm{m}$. The included individuals agreed to have CBCT for their MFMs teeth for research purposes.

\section{Recorded features}

After recording gender and tooth position, the following anatomic features of teeth were determined, characterized and recorded for each molar by carefully rolling downward in a one $\mathrm{mm}$ distance through the CBCT images from the pulp chamber floor to the root's apex at the axial tomographic slices:

\section{a. External morphology}

- Number of roots

- Type of roots (separated or fused)

- Morphology of root: the shape was classified as one of the basic seven configurations of root cross-section outlines (round, oval, long oval, ribbon-shaped, kidney-shaped, bowling pin or hourglass).

b. Internal morphology

- Number of canals orifices per each root

- Type of canals in each root according to Vertucci's classification (2) which include eight different types.

c. Primary variations in morphology of root (external) and root canal systems (internal) according to Zhang et al. variants (6). These are ten variants describing the different numbers and types of roots and the different number of canals in each root(s).

After calibration of the researchers based on the anatomical criteria and variants used in this study, all images were evaluated by two endodontists simultaneously. In case of disagreement in analysis and interpretation of the radiographic findings, a consensus was reached after a discussion between the two endodontists. CBCT slices for each tooth were analyzed 
using Ez3D Plus image software (VATECH Global, Korea). Axial, sagittal, and coronal two-dimensional sections of each scan were displayed and data were recorded. Whenever necessary, image contrast and brightness were adjusted using image processing tool in the used software in order to achieve best display and visualization.

\section{Statistical analysis}

Recorded data were collected, tabulated and statistically analyzed using SPSS software for Windows version 21.0 (SPSS Inc., Chicago, IL, USA). Prevalence of number of roots and their types, root shape in cross-section, number of canal orifices within each root, type of canal configuration within each root and root and root canal variant were calculated. Chi-square test and Fisher's Exact test were used to determine the significant differences of the study parameters between females and males and left and right sides. $P$ value of $\leq 0.05$ was considered statistically significant.

\section{RESULTS}

\section{Number and type of roots}

Table 1 summarizes the number of roots in relation to gender and tooth position on right and left sides. MFMs had mostly two roots in $96.8 \%(n=484)$ and only $3.2 \%(n=16)$ with RE (Fig. 1). Bilateral occurrence of same number of roots (whether two or three roots) on both sides was found in $96.8 \%(n=242)$ of individuals and $3.2 \%$ (four males and four females) showed different numbers of roots of right and left MFMs.

Three-rooted MFMs were found in females $(n=12,4.8 \%)$ more than males ( $n=4,1.6 \%)$ with statistically significant difference of $P<0.05$. RE DL root was bilateral in four females $(n=8)$ and unilateral in eight individuals without preference to any side of the mouth over the other. All molars had separate roots and no fused roots observed.

\section{Root cross-section}

Mesial root was mainly ribbon-shaped in cross-section $(92.2 \%$, $n=461)$. Kidney, long oval, and bowling pin shapes were observed in lower percentages (4.6\%, 3\%, and $0.2 \%$ respectively). Distal root was mainly kidney-shaped $(56.2 \%, n=281)$ or long oval (31.4\%, $n=157)$. Bowling pin, ribbon and oval shapes were also found in $4.4 \%, 4.2 \%$, and $3.8 \%$ respectively. In threerooted molars, RE DL root was mainly round in cross-section $(2.8 \%, n=14)$ and oval in two molars $(0.4 \%)$. Examples of root cross-section shapes that were found in this study are shown in Figure 2.

TABLE 1. Number of roots and prevalence of RE for gender and tooth position

\begin{tabular}{|c|c|c|c|c|c|c|}
\hline & \multicolumn{3}{|c|}{ Gender } & \multicolumn{3}{|c|}{ Tooth position } \\
\hline & Male & Female & Total & Right side & Left side & Total \\
\hline Two-rooted molars n (\%) & $246(49.2)$ & $238(47.6)$ & $484(96.8)$ & $242(48.4)$ & $242(48.4)$ & $484(96.8)$ \\
\hline Three-rooted molars n (\%) & $4(0.8)$ & $12(2.4)^{*}$ & $16(3.2)$ & $8(1.6)$ & $8(1.6)^{\dagger}$ & $16(3.2)$ \\
\hline Total n (\%) & $250(50)$ & $250(50)$ & $500(100)$ & $250(50)$ & $250(50)$ & $500(100)$ \\
\hline
\end{tabular}

${ }^{*} \mathrm{P}$ value $=0.042(<0.05),{ }^{\mathrm{P}} \mathrm{P}$ value $=1.000(>0.05)$
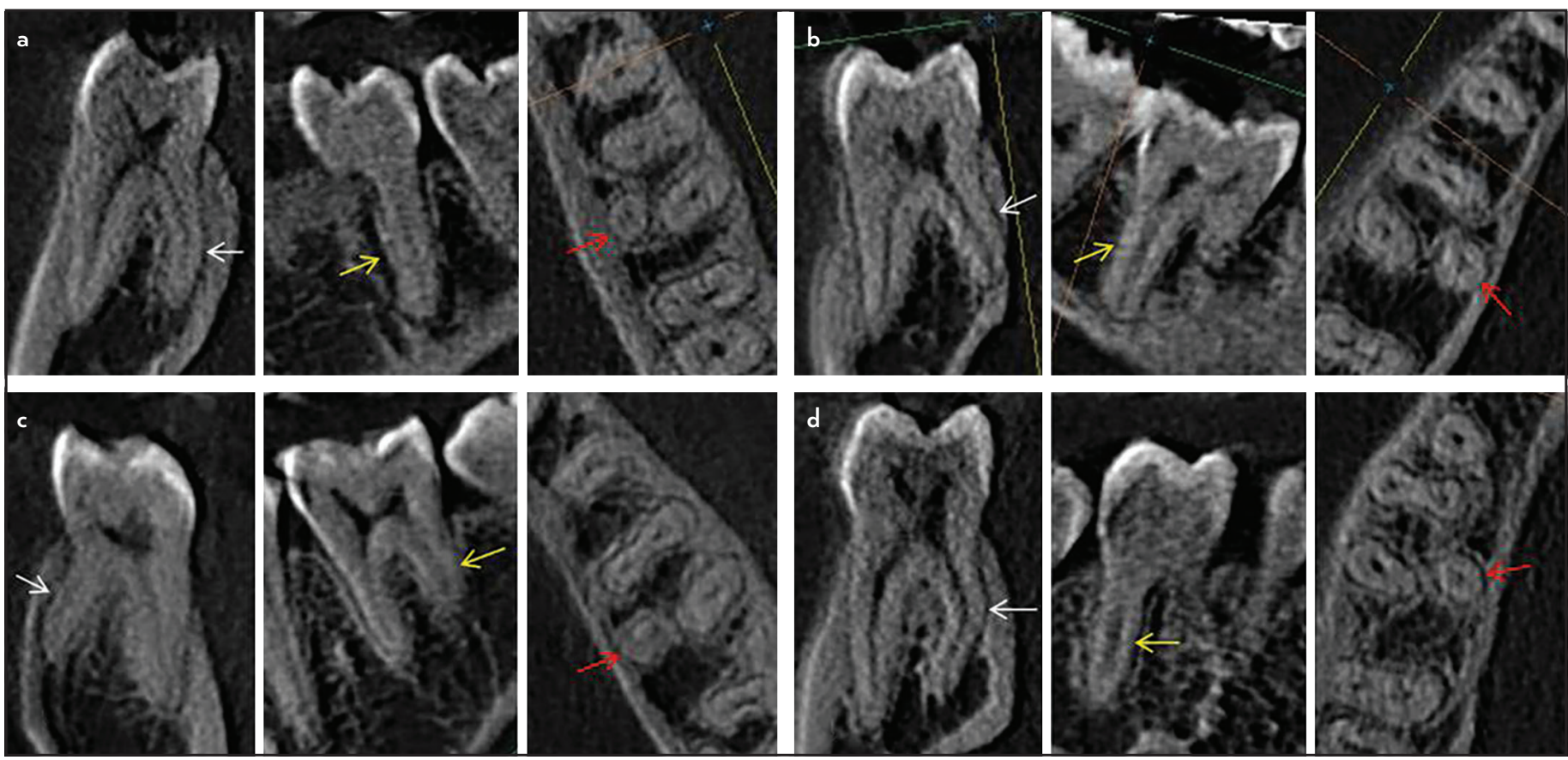

Figure 1. Examples of Radix Entomolaris found in this study (white arrows in sagittal plan, yellow arrows in coronal plan, and red arrows in axial plan). (a) Female left side, (b) Female right side, (c) Male left side, and (d) Male right side 


\section{Number of root canal orifices}

Table 2 shows total number of canals orifices in molars included in this study. Three canals orifices are the most common type in two-rooted molars $(89.4 \%, \mathrm{n}=447)$ where mesial root displayed two canals orifices (95.8\%) and distal root showed one canal orifice (96.4\%). In addition, there were molars with two root canals orifices (3.6\%) and four root canals orifices (3.4\%). All three-rooted molars have four canals orifices (3.2\%), two in the mesial root, one in the distal root and one in the RE.

Bilateral similarity of canals orifices number on both sides of oral cavity occurred in $94.8 \%(n=237)$ for mesial root and in $93.6 \%$ $(n=234)$ for distal root. Considering the total number of canals orifices per each molar, $86.8 \%(n=217)$ showed bilateral equal number of canals orifices of both right and left molars. The frequency distribution of the total number of canals orifices did not differ significantly between females and males ( $P>0.05)$.

\section{Root canal configurations}

Variations in the root canal types according to Vertucci's classification were observed in mesial, distal and DL roots. In mesial root, type II was the most frequent in both gender, accounting for $57 \%$ of the sample $(n=285)$, followed by type IV $(35.6 \%$, $\mathrm{n}=178)$. In distal root, type III was the most prevalent $(48.8 \%$, $n=244)$, followed by type I $(41 \%, n=205)$. The prevalence of Vertucci's remaining types, additional types of Vertucci (Sert and Bayirli (25) additional types and Gulabivala et al. (7) additional type) and other types not included in his classification nor in additional types are shown in Table 3. RE roots showed type I canal type. Bilateral similarity of the canal type in both sides of oral cavity was $67.6 \%(n=169)$ in mesial roots and $63.2 \%$ $(n=158)$ in distal root. The rest showed different canal types of roots on the right side from the left side. No significant differences of root canal types were found between males and females or between right and left sides.

\section{Primary variations in the morphology of root and root canal systems}

According to Zhang et al. (6), only variants 1, 3, 4 and 6 were observed (Table 4). The presence of two canals in the mesial root and one canal in the distal root of teeth with two separate
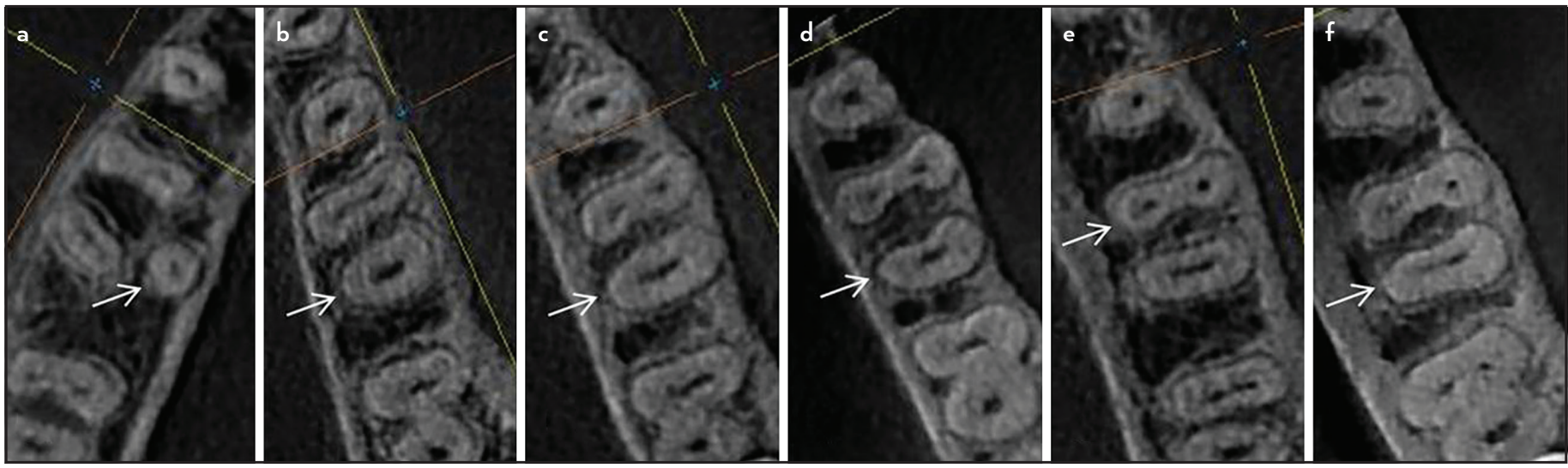

Figure 2. Examples of root cross-section outlines of MFMs (white arrows): (a) Round, (b) Oval, (c) Long oval, (d) Kidney-shaped, (e) Ribbonshaped and ( $f$ ) Bowling pin

TABLE 2. Number of root canals orifices

\begin{tabular}{lcccccc}
\hline & \multicolumn{3}{c}{ Total number of root canals orifices per tooth } & \multicolumn{2}{c}{ Total } \\
\cline { 2 - 5 } & Two & Three & Four & Five & Six & $\mathrm{n}(\%)$ \\
\hline Two-rooted molars $\mathrm{n}(\%)$ & $18(3.6)$ & $447(89.4)$ & $17(3.4)$ & $1(0.2)$ & $1(0.2)^{*}$ & $484(96.8)$ \\
Three-rooted molars $\mathrm{n}(\%)$ & 0 & 0 & $16(3.2)$ & 0 & $16(3.2)$ & 0 \\
Total $\mathrm{n}(\%)$ & $18(3.6)$ & $447(89.4)$ & $33(6.6)$ & $1(0.2)$ & $1(0.2)$ & $500(100)$ \\
\hline
\end{tabular}

*P value $=0.001(<0.05)$

TABLE 3. Distribution of root canal types according to Vertucci's classification, other additional types and new types

\begin{tabular}{|c|c|c|c|c|c|c|c|c|c|c|c|c|c|}
\hline & \multirow[b]{2}{*}{1} & \multicolumn{7}{|c|}{ Vertucci's canal types } & \multicolumn{3}{|c|}{ Vertucci's additional types } & \multirow{2}{*}{$\frac{\text { Other types* }}{2-3-2-1}$} & \multirow{2}{*}{ Tota } \\
\hline & & II & III & IV & V & $\mathrm{Vl}$ & VII & VIII & $2-3-2^{+}$ & $3-2^{\ddagger}$ & $1-2-3-2^{+}$ & & \\
\hline \multicolumn{14}{|c|}{ Mesial root } \\
\hline$n$ & 4 & 285 & 11 & 178 & 2 & 5 & 0 & 2 & 9 & 1 & 0 & 3 & 500 \\
\hline$\%$ & 0.8 & 57 & 2.2 & 35.6 & 0.4 & 1 & 0 & 0.4 & 1.8 & 0.2 & 0 & 0.6 & 100 \\
\hline \multicolumn{14}{|c|}{ Distal root } \\
\hline$n$ & 205 & 12 & 244 & 4 & 25 & 1 & 6 & 1 & 1 & 0 & 1 & 0 & 500 \\
\hline$\%$ & 41 & 2.4 & 48.8 & 0.8 & 5 & 0.2 & 1.2 & 0.2 & 0.2 & 0 & 0.2 & 0 & 100 \\
\hline
\end{tabular}

${ }^{\dagger}$ Sert and Bayirli additional types, ${ }^{\ddagger}$ Gulabivala et al. additional type, ${ }^{*}$ Not included in Vertucci's classification or within the additional types 
roots (variant 3) represented the most common morphology $(89.8 \%)$. Variant 1, variant 4, and variant 6 were also found in equal percentages (3.2\% each) (Fig. 3). A percentage of 0.6 $(n=3)$ was not included within the ten variants of Zhang et al. These three molars were found in males on the right side; all have two separated roots with three canals in the mesial root but one with one canal in the distal root, second with two canals in the distal root and third with three canals in the distal root (Fig. 4). No significant difference was found between males and females or between right and left sides ( $P>0.05)$. Regarding the occurrence of the same variant on both sides of oral cavity, only $13.2 \%(n=33)$ showed different variants on both sides, while the majority $(86.8 \%, \mathrm{n}=217)$ showed similar variants on both sides.

\section{DISCUSSION}

Several studies have been carried out among different populations around the world to investigate possible differences re-

TABLE 4. Distribution of Zhang et al. variants of root and canal system morphology

\begin{tabular}{|c|c|c|c|c|c|c|c|c|c|c|c|c|}
\hline & \multicolumn{10}{|c|}{ Variant } & $\begin{array}{l}\text { Not included } \\
\text { "New Variant" }\end{array}$ & Total \\
\hline Percentage & 3.2 & 0 & 89.8 & 3.2 & 0 & 3.2 & 0 & 0 & 0 & 0 & 0.6 & 100 \\
\hline
\end{tabular}
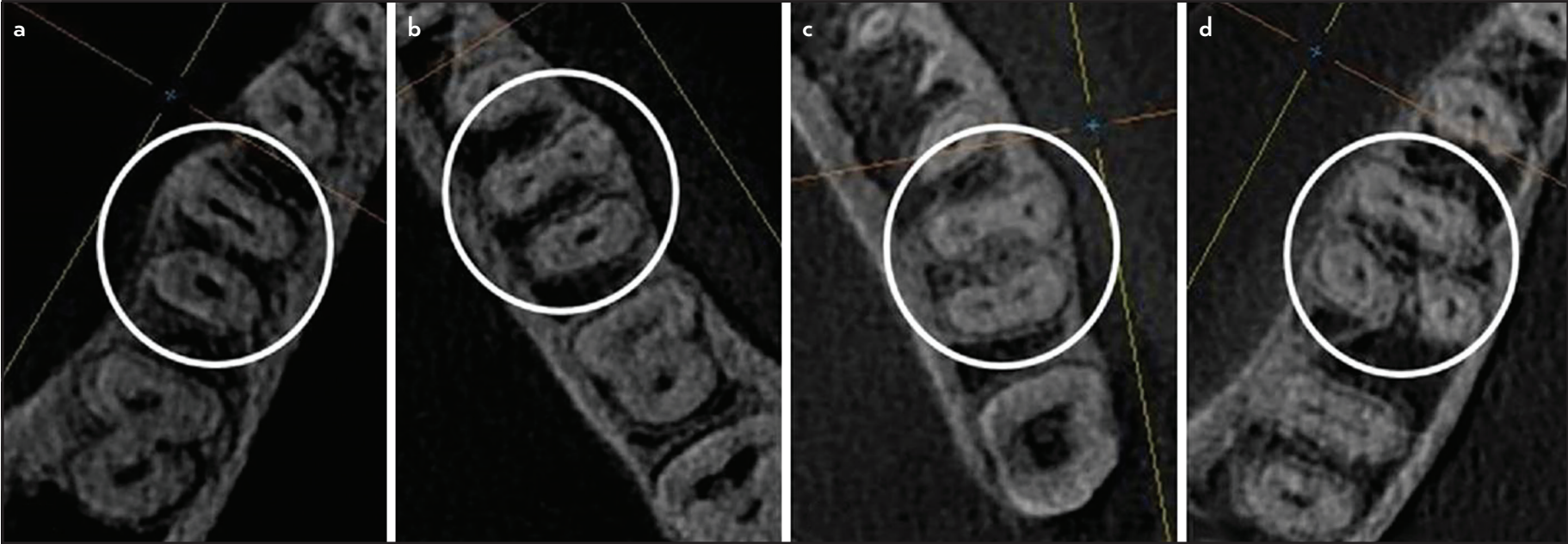

Figure 3. Examples of Zhang et al. variants of roots and root canals that were found in mandibular first molars in this study (white circles): (a) variant 1, (b) variant 3 , (c) variant 4 and (d) variant 6

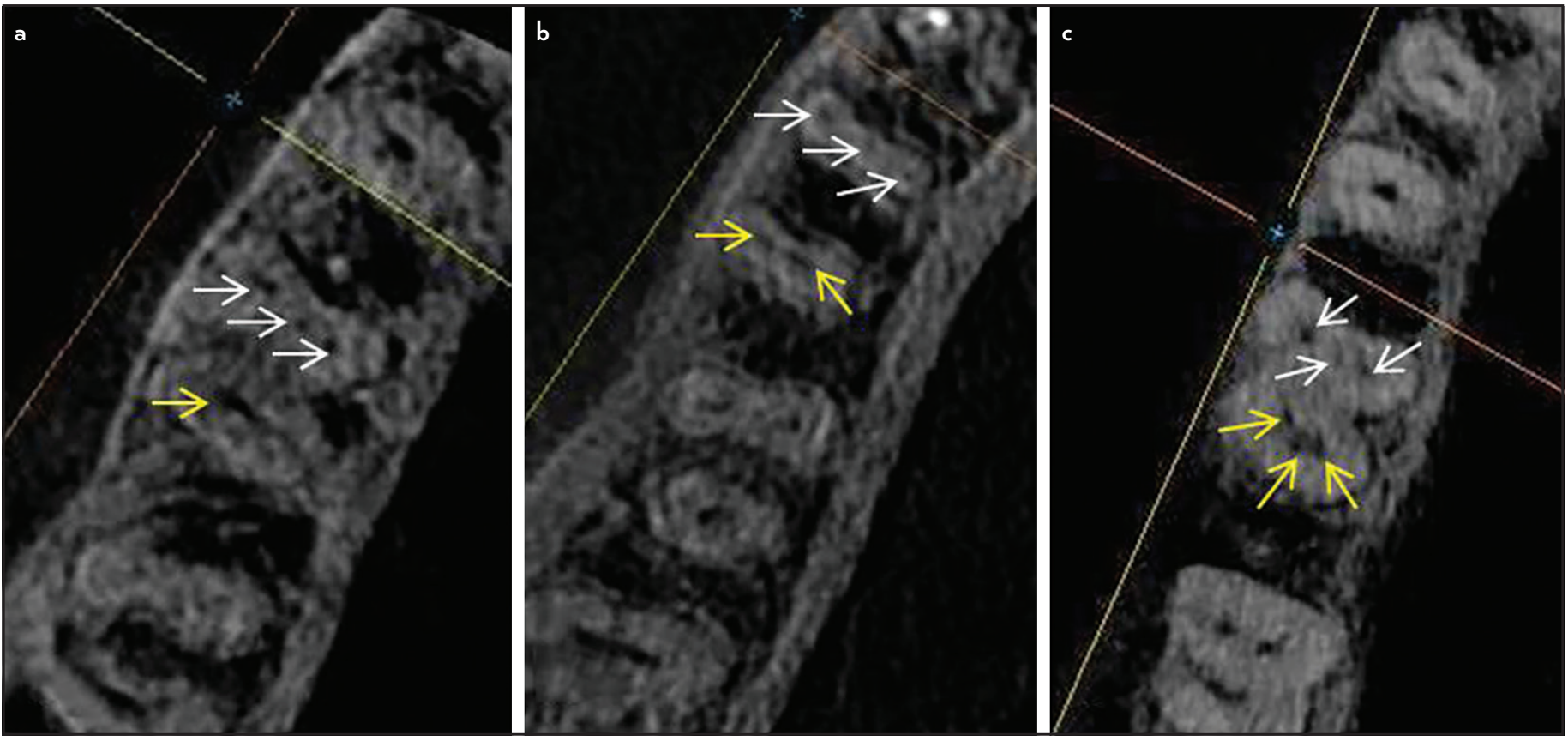

Figure 4. Three molars that were not included within Zhang et al. variants; all have two separated roots with three canals in the mesial root and (a) one canal, (b) two canals and (c) three canals in the distal root (white arrows pointing mesial root canals and yellow arrows pointing distal root canals) 
lated to race and geographical regions in the internal anatomy of MFMs $(1,3,4,6-12)$. The current study is the first that concerned root configuration and root canal morphology of these teeth in a Yemeni population.

The mean age of the sample included in this study was $24.2 \pm 4.7$ (for males: $23.7 \pm 4.8$, for females: $24.6 \pm 4.5$ ). Age effects on internal anatomy were not considered in this study because only individuals with no or minimum aging effects on their teeth canal anatomy were included. In addition, only sound MFMs or those with minimal non-cavitation caries were included to minimize any consequent effects of dentine deposition on pulp anatomy.

Various methods have been proposed to determine the morphologic characteristics of the root canal system. Even though clearing technique is used since a long time, however, it is a destructive and non-reproducible technique that might create artifacts when used to identify the root canal configuration and can only be used in vitro (21). This may not accurately reflect the actual root canal morphology due to failure of dye to penetrate and stain the whole root canal system in cleared teeth particularly in Vertucci type I canal configuration (21). Although these limitations have been overcome by micro-CT with higher resolution and accuracy providing detailed quantitative and qualitative measurements of root canal anatomy, nevertheless, micro-CT has limited availability around the world specifically in underdeveloped and developing countries. Furthermore, its high cost and radiation dose are other factors for its use only in vitro (21). On the other hand, CBCT technique has been used to evaluate root and root canal anatomy $(15,26)$. It provides a practical tool for noninvasive and three-dimensional reconstruction imaging for clinicians in teeth morphological analysis and different endodontic applications (27). CBCT has shown its reliability and accuracy in revealing the number and of root canals compared to the used standard methods such as clearing and micro-CT techniques (21). Moreover, CBCT is an available and less expensive method that can be used either in vivo or ex vivo. Therefore, it was selected in the present study for evaluation of root and root canal configuration of MFMs in a Yemeni population. Additionally, the use of CBCT images in the present study facilitated the assessment of molars without teeth destruction compared to other methods.

Vertucci's classification (2) was taken as a reference for canal types in the present study because it is the first system that identified complex canal system configurations than previous classification. Even though it is a basic classification since a long period of time, however, it is still commonly used in recent studies by most authors in the literature and textbooks $(15,21)$ and it was used in the present study for easier comparison with other studies' results. Additionally, the classification proposed and published by Zhang et al. (6) was used in this study because it represents a comprehensive classification that correlates the number of roots to the number of root canals of each tooth and giving a simple description of tooth internal and external anatomy.

$\mathrm{RE}$ is considered as an anatomical variation than a developmental anomaly (28) with varying prevalence among different populations. The incidence of RE in the studied population was $3.2 \%$. This is similar to previous studies in Sudanese (3\%) (10) and Belgians (3\%) (8). In addition, the low percentage of RE coincided with the low incidence (not more than 5-6\%) reported in studies of non-Asian populations $(1,2,8,18)$. However, our finding was lower than that of Asian populations who showed higher percentages as 10\% in Burmese (7), 13\% in Thai (4), and $25.61 \%$ in Taiwanese (27). The highest percentage in Asian population was reported in Chinese that ranged between $22.1 \%$ and $29 \%(6,15)$. The occurrence of RE root in the present study was higher than the findings of studies in Turkey (14) and Iran (17) where RE was only found in $0.5 \%$ and $1.9 \%$ respectively. Additionally, earlier studies on Brazilians (26) and Pakistanis (16) showed no three-rooted MFMs.

RE was observed in a significantly higher percentage in females than males. This finding does not agree with previous studies in Taiwan (27), Germany (9), and Iran (18) which showed no differences in RE DL occurrence in relation to gender. RE was found bilaterally and unilaterally with no preference to right or left sides which is similar to what was found in a previous study (29). However, Schäfer et al. (9) found three-rooted molars in their study only occurred unilaterally with no significant difference in the side of occurrence. Dastgerdi et al. (18) found only bilateral occurrence of RE in $5.1 \%$ of their sample. Tu et al. (27), Choi et al. (29), and Zhang et al. (15) studies revealed that $\mathrm{RE}$ root occurred more frequently on the right side than on the left. Conversely, another study showed a more frequent occurrence of RE on the left side than on the right (7). Studies' variations in methods used for investigation of molars internal anatomy, sample size and different populations can explain those controversial results (9).

Despite the low percentage of RE found in this study, it should be expected and looked for as a form of unusual root variation of these molars (5). Using angled preoperative periapical radiographs or CBCT help evaluating teeth anatomy, thus, avoiding missed canals. Modification of access opening in case of presence of RE by extension of cutting in a DL direction is necessary to locate RE canal orifice and create a straight line access (5).

No RP root was found in this study. This is similar to the findings in Sudanese (10), Turkish population (12), Iranians (18) and Taiwanese (27).

Fused roots were not found in this study, which was in agreement with a previous study in Sudanese (10). However, Demirbuga et al. (12) reported a $1.7 \%$ of root fusion in MFMs in Turkey. Moreover, a higher percentage (19.1\%) of root fusion was recorded in Thai MFMs (4).

The mesial root was mainly ribbon-shaped in cross-section and the distal root was kidney-shaped. This emphasizes the essential care required during canals enlargement of such molars so that procedural errors such as perforations can be avoided in danger zones of thin dentine due to anatomical depressions and grooves of roots' surfaces (30).

Mesial root showed two canals orifices in $95.8 \%$ which is higher than found in previous study in Sudan (86\%). On the other hand, distal root had mainly one canal orifice (96.4\%) 
and showed two canals orifices in only $3.4 \%$ which is much lower than reported in Sudanese (10) where distal root showed two canals orifices in 59\% and another study in Iranians (18) where distal root had two canals orifices in $52.9 \%$. Differences in these findings may be related to racial issues.

Diverse Vertucci root canal configurations of MFMs were found in this study. In mesial root, type II was the dominant type $(57 \%)$ followed by type IV $(35.6 \%)$, which was consistent with the findings of earlier studies $(19,20)$. Nevertheless, the findings of this study disagreed with other studies' results where type IV was the most prevalent type followed by type II $(10,12,14-18)$. In distal root, type III was the most prevalent (48.8\%), followed by type I (41\%). However, this is inconsistent with other previous investigations where type I was the dominant type and type III was reported in none or lower percentages $(8,10,12,14-18)$.

Vertucci canal types I, II, IV, and VIII are relatively easier to be identified, prepared and obturated than other types with a more complex anatomy including multiple canals with canals fusion or divisions along their course within the root such as Vertucci canal types III, V, VI, and VII, additional types of Vertucci and other types not included in Vertucci's classification or within the additional types were found in this study. Despite their low percentages, failure to properly debride such complex canal anatomy might affect treatment outcome. Vertucci canal type VIII was found in a low percentage $(0.4 \%$ in mesial root and $0.2 \%$ in distal root) close to previous study results (14). A higher percentage of Vertucci canal type VIII was reported by Ahmed et al. (10) in Sudanese, while other studies' samples found none $(12,16)$. No C-shaped roots and canals were observed in the present study compared to previous studies where lower percentages were found in MFMs of other populations: $0.85 \%$ in Turkish (12), $1.2 \%$ in Iranians (17), and $1.7 \%$ in Brazilians (26).

Types of canal in this study (type 2-3-2-1) which is not included in Vertucci's classification or its additional types can be classified according to the new classification system suggested by Ahmed et al. (31). This classification system uses three-coded formula to describe tooth numbering, number of roots, and canal configuration in each root. The new formula can be used to describe this type of canal (type 2-3-2-1) found in the mesial root in three MFMs in males. Two of them (one on the left side and one on the right side) have Vertucci type I canal in their distal roots and one (on the right side) with Vertucci type III canal in its distal root. According to this new classification system, those three MFMs will be codded as: ${ }^{2} 36 \mathrm{M}^{2-3-2-1} \mathrm{D}^{1},{ }^{2} 46$ $M^{2-3-2-1} D^{1}$ and ${ }^{2} 46 M^{2-3-2-1} D^{1-2-1}$, respectively.

Of the 500 MFMs included in our study, 449 molars (89.8\%) had two separate roots with three canals (variant 3 ). This result was in the same line with previous reports $(1,3,4,7,15)$. Variant 1 was found in the study in $3.2 \%$, which was higher than reported by Zhang et al. (0.5\%) in Chinese (6). However, variant $4(3.2 \%)$ and variant $6(3.2 \%)$ reported in this study were lower than reported by the same authors where variants 4 and 6 were $12 \%$ and $21.8 \%$, respectively (6). Only three molars $(0.6 \%)$ showed other variants not included within Zhang et al. (6) variants, all were found in males.
Findings of the current study provided some information about root and canal anatomy of the MFMs in a Yemeni population and confirmed the presence of anatomical differences among various populations. Moreover, despite the low percentages of RE and of complex canal configurations, it is still necessary to perform a careful clinical and radiographical examination during root canal treatment of these teeth to avoid any missed canals, promote success and improve outcome. Additionally, further investigations for the anatomy of other teeth in Yemen are required to establish a base line data and compare findings with those of other populations.

\section{CONCLUSION}

The double-rooted mandibular molar is the most common variant with $3.2 \%$ of Yemeni MFMs have an extra RE with a higher occurrence in females than males. The mesial roots are ribbon-shaped and the distal roots are kidney-shaped in crosssection. Vertucci type II and III canal configurations showed the higher incidence in mesial and distal roots, respectively. In addition, Zhang et al. variant 3 was the most common morphology.

\section{Disclosures}

Conflict of interest: The authors deny any conflict of interest.

Ethics Committee Approval: Ethics committee approval was received for this study from the Medical Ethics Committee of Faculty of Medicine and Health Sciences at University of Science and Technology (UST), Sana'a, Yemen (MECA NO.: EAC/UST138).

Peer-review: Externally peer-reviewed.

Financial Disclosure: The authors declared that this study has received no financial support.

Authorship contributions: Concept - E.M.S., H.A.A.; Design - E.M.S., A.A.M., H.A.A.; Supervision - A.A.M., H.A.A.; Funding - E.M.S.; Materials - E.M.S.; Data collection \&/or processing - E.M.S., A.A.M.; Analysis and/or interpretation E.M.S., A.A.M., H.A.A.; Literature search - E.M.S., A.A.M., H.A.A.; Writing - E.M.S., A.A.M., H.A.A.; Critical Review - E.M.S., A.A.M., H.A.A.

\section{REFERENCES}

1. de Pablo OV, Estevez R, Péix Sánchez M, Heilborn C, Cohenca N. Root anatomy and canal configuration of the permanent mandibular first molar: a systematic review. J Endod 2010; 36(12):1919-31.

2. Vertucci FJ. Root canal anatomy of the human permanent teeth. Oral Surg Oral Med Oral Pathol 1984; 58(5):589-99.

3. Chandra SS, Chandra S, Shankar P, Indira R. Prevalence of radix entomolaris in mandibular permanent first molars: a study in a South Indian population. Oral Surg Oral Med Oral Pathol Oral Radiol Endod 2011; 112(3):e77-e82.

4. Gulabivala K, Opasanon A, Ng YL, Alavi A. Root and canal morphology of Thai mandibular molars. Int Endod J 2002; 35(1):56-62.

5. Calberson FL, De Moor RJ, Deroose CA. The radix entomolaris and paramolaris: clinical approach in endodontics. J Endod 2007; 33(1):58 63.

6. Zhang R, Wang H, Tian YY, Yu X, Hu T, Dummer PM. Use of cone-beam computed tomography to evaluate root and canal morphology of mandibular molars in Chinese individuals. Int Endod J 2011;44(11):990-9.

7. Gulabivala $\mathrm{K}$, Aung TH, Alavi A, Ng YL. Root and canal morphology of Burmese mandibular molars. Int Endod J 2001; 34(5):359-70.

8. Torres A, Jacobs R, Lambrechts P, Brizuela C, Cabrera C, Concha G, et al. Characterization of mandibular molar root and canal morphology using cone beam computed tomography and its variability in Belgian and Chilean population samples. Imaging Sci Dent 2015; 45(2):95-101.

9. Schäfer E, Breuer D, Janzen S. The prevalence of three-rooted mandibular permanent first molars in a German population. J Endod 2009; 35(2):202-5 
10. Ahmed HA, Abu-bakr NH, Yahia NA, Ibrahim YE. Root and canal morphology of permanent mandibular molars in a Sudanese population [published correction appears in Int Endod J. 2007 Dec;40(12):1008]. Int Endod J 2007; 40(10):766-71.

11. Ahmad IA. Root and root canal morphology of Saudi Arabian permanent dentition. Saudi Endod J 2015; 5(2):99-106.

12. Demirbuga S, Sekerci AE, Dinçer AN, Cayabatmaz M, Zorba YO. Use of cone-beam computed tomography to evaluate root and canal morphology of mandibular first and second molars in Turkish individuals. Med Oral Patol Oral Cir Bucal 2013; 18(4):e737-e44.

13. De Moor RJ, Deroose CA, Calberson FL. The radix entomolaris in mandibular first molars: an endodontic challenge. Int Endod J 2004; 37(11):789-99.

14. Nur BG, Ok E, Altunsoy M, Aglarci OS, Colak M, Gungor E. Evaluation of the root and canal morphology of mandibular permanent molars in a south-eastern Turkish population using cone-beam computed tomography. Eur J Dent 2014; 8(2):154-9.

15. Zhang X, Xiong S, Ma Y, Han T, Chen X, Wan F, et al. A Cone-Beam Computed Tomographic Study on Mandibular First Molars in a Chinese Subpopulation. PLoS One 2015; 10(8):e0134919.

16. Faraz SA, Tariq A, Jameel A. Root canal morphology of mandibular first permanent molars-Karachi sample. Pakistan Oral Dent J 2015; 35(2):294-8.

17. Madani ZS, Mehraban N, Moudi E, Bijani A. Root and Canal Morphology of Mandibular Molars in a Selected Iranian Population Using Cone-Beam Computed Tomography. Iran Endod J 2017; 12(2):143-8.

18. Destgerdi AC, Navabi M, Hafezi L, Khalilak Z, Rakhshan V. Anatomy of Permanent Mandibular First Molars in a Selected Iranian Population Using Cone-beam Computed Tomography. Iran Endod J 2018; 13(2):251-6.

19. Zaatar El, al Anizi SA, al Duwairi Y. A study of the dental pulp cavity of mandibular first permanent molars in the Kuwaiti population. J Endod 1998; 24(2):125-7.

20. al-Nazhan S. Incidence of four canals in root-canal-treated mandibular first molars in a Saudi Arabian sub-population. Int Endod J 1999; 32(1):49-52.
21. Ordinola-Zapata R, Bramante CM, Versiani MA, Moldauer BI, Topham G, Gutmann JL, et al. Comparative accuracy of the Clearing Technique, CBCT and Micro-CT methods in studying the mesial root canal configuration of mandibular first molars. Int Endod J 2017; 50(1):90-6.

22. Nair MK, Nair UP. Digital and advanced imaging in endodontics: a review. J Endod 2007; 33(1):1-6.

23. Chaloob EK. Oral health status, dental knowledge and behaviors among children and adolescents (8-15) years old in the cities of Baghdad and Thamar. J Baghdad Coll Den 2013; 25(4):100-3.

24. Mukhaimer RH. Evaluation of Root Canal Configuration of Mandibular First Molars in a Palestinian Population by Using Cone-Beam Computed Tomography: An Ex Vivo Study. Int Sch Res Notices 2014; 2014:583621.

25. Sert S, Bayirli GS. Evaluation of the root canal configurations of the mandibular and maxillary permanent teeth by gender in the Turkish population. J Endod 2004; 30(6):391-8.

26. Silva EJ, Nejaim Y, Silva AV, Haiter-Neto F, Cohenca N. Evaluation of root canal configuration of mandibular molars in a Brazilian population by using cone-beam computed tomography: an in vivo study. J Endod 2013; 39(7):849-52.

27. Tu MG, Huang HL, Hsue SS, Hsu JT, Chen SY, Jou MJ, et al. Detection of permanent three-rooted mandibular first molars by cone-beam computed tomography imaging in Taiwanese individuals. J Endod 2009; 35(4):503-7.

28. Walker RT. Root form and canal anatomy of mandibular first molars in a southern Chinese population. Endod Dent Traumatol 1988; 4(1):19-22.

29. Choi MR, Moon YM, Seo MS. Prevalence and features of distolingual roots in mandibular molars analyzed by cone-beam computed tomography. Imaging Sci Dent 2015; 45(4):221-6.

30. Zhou G, Leng D, Li M, Zhou Y, Zhang C, Sun C, et al. Root dentine thickness of danger zone in mesial roots of mandibular first molars. BMC Oral Health 2020; 20(1):43.

31. Ahmed HMA, Versiani MA, De-Deus G, Dummer PMH. A new system for classifying root and root canal morphology. Int Endod J 2017; 50(8):761-70. 